DTP $/ 00 / 12$

February 2000

\title{
Complete Renormalization Group Improvement- Avoiding Factorization and Renormalization Scale Dependence in QCD Predictions
}

\author{
C.J. Maxwell ${ }^{1)}$ and A. Mirjalili2) \\ Centre for Particle Theory, University of Durham \\ South Road, Durham, DH1 3LE, England
}

\begin{abstract}
For moments of leptoproduction structure functions we show that all dependence on the renormalization and factorization scales disappears provided that all the ultraviolet logarithms involving the physical energy scale $Q$ are completely resummed. The approach is closely related to Grunberg's method of Effective Charges. A direct and simple method for extracting $\Lambda_{\overline{M S}}$ from experimental data is advocated.
\end{abstract}

1) C.J.Maxwell@durham.ac.uk

2) Abolfazl.Mirjalili@durham.ac.uk 


\section{Introduction}

The problem of renormalization scheme dependence in QCD perturbation theory remains on obstacle to making precise tests of the theory. In a recent paper [1] one of us pointed out that the renormalization scale dependence of dimensionless physical QCD observables, depending on a single energy scale $Q$, can be avoided provided that all ultraviolet logarithms which build the physical energy dependence on $Q$ are resummed. This was termed complete Renormalization Group ( $\mathrm{RG}$ )-improvement in Ref.[1]. It was stressed that standard RG-improvement, as customarily applied with a $Q$-dependent scale $\mu=x Q$, omits an infinite subset of these logarithms. One should rather keep $\mu$ independent of $Q$, and then carefully resum to all-orders the RG-predictable ultraviolet logarithms. In this way all $\mu$-dependence cancels between the renormalized coupling and the logarithms of $\mu$ contained in the coefficients, and the correct physical $Q$-dependence is built. At next-to-leading order (NLO) the result is identical to the Effective Charge approach of Grunberg [2, 3]. We wish to extend this argument to processes involving factorization of operator matrix elements and coefficient functions, where a factorization scale $M$ arises in addition to the renormalization scale $\mu$. We shall use the prototypical factorization problem of moments of leptoproduction structure functions as a specific example. We shall identify the logarithms of $\mu, M$, and $Q$ which occur, and will show explicitly that on resumming all the ultraviolet logarithms the $\mu$ and $M$ dependence disappears. We shall organize the paper so that we review the treatment of Ref.[1] whilst showing how it generalizes for the moment problem. We begin in Section 2 by giving some basic definitions for the moments of structure functions. Section 3 considers the dependence of the perturbative coefficients on the parameters which label the renormalization procedure in both cases. Section 4 deals with the complete RG-improvement of the structure function moments and identifies and resums the physical ultraviolet logarithms. Finally, in Section 5 we discuss a more straightforward way of motivating this approach, and consider how to directly extract $\Lambda_{\overline{M S}}$ from data. We also give our Conclusions. 


\section{Structure Function Moments}

In the prototypical factorization problem of deep inelastic leptoproduction the $n^{\text {th }}$ moment of a non-singlet structure function $F(x)$,

$$
\mathcal{M}_{n}(Q)=\int_{0}^{1} x^{n-2} F(x) d x
$$

can be factorized in the form

$$
\mathcal{M}_{n}(Q)=<\mathcal{O}_{n}(M)>\mathcal{C}_{n}(Q, a(\mu), \mu, M)
$$

Here $M$ is an arbitrary factorization scale and $a(\mu)$ is the RG-improved coupling $\alpha_{s}(\mu) / \pi$ defined at a renormalization scale $\mu$. The operator matrix element $<\mathcal{O}_{n}(M)>$ has an $M$-dependence given by its anomalous dimension,

$$
\frac{M}{<\mathcal{O}>} \frac{\partial<\mathcal{O}>}{\partial M}=\gamma_{\mathcal{O}}(a)=-d a-d_{1} a^{2}-d_{2} a^{3}-d_{3} a^{4}+\ldots
$$

For simplicity we shall from now on suppress the $n$-dependence of terms in equations, as we have done in Eq.(3). For a given moment $d$ is independent of the factorization convention, whereas the higher $d_{i},(i \geq 1)$ depend on it. In Eq.(3) the coupling $a$ is governed by the $\beta$-function equation

$$
M \frac{\partial a}{\partial M}=\beta(a)=-b a^{2}\left(1+c a+c_{2} a^{2}+c_{3} a^{3}+\ldots\right) .
$$

Here $b=\left(33-2 N_{f}\right) / 6$, and $c=\left(153-19 N_{f}\right) / 12 b$, are the first two coefficients of the beta-function for SU(3) QCD with $N_{f}$ active flavours of quark. They are universal, whereas the subsequent coefficients $c_{2}, c_{3}, \ldots$ are scheme-dependent. Equation (3) can be integrated to [甘, 5]

$$
<\mathcal{O}(M)>=A \exp \left[\int_{0}^{a} \frac{\gamma(x)}{\beta(x)} d x-\int_{0}^{\infty} \frac{\gamma^{(1)}(x)}{\beta^{(2)}(x)} d x\right],
$$

where $\gamma^{(1)}$ and $\beta^{(2)}$ denote these functions truncated at one and two terms, respectively. The factor $A$ is scheme-independent [5] and can be fitted to experimental data. The second integral in Eq.(5) is an infinite constant of 
integration. In Eq. (2) $\mathcal{C}(Q, a(\mu), \mu, M)$ is the coefficent function and has the perturbation series

$$
\mathcal{C}(Q, \tilde{a}, \mu, M)=1+r_{1} \tilde{a}+r_{2} \tilde{a}^{2}+r_{3} \tilde{a}^{3}+\ldots
$$

We shall use $\tilde{a}$ to stand for $a(\mu)$ and $a$ for $a(M)$. After combining the integrals in Eq.(5) one obtains

$$
\mathcal{M}=A\left(\frac{c a}{1+c a}\right)^{d / b} \exp (\mathcal{I}(a))\left(1+r_{1} \tilde{a}+r_{2} \tilde{a}^{2}+r_{3} \tilde{a}^{3}+\ldots\right),
$$

where $\mathcal{I}(a)$ is the finite integral

$$
\mathcal{I}(a)=\int_{0}^{a} d x \frac{d_{1}+\left(d_{1} c+d_{2}-d c_{2}\right) x+\left(d_{3}+c d_{2}-c_{3} d\right) x^{2}+\ldots}{b(1+c x)\left(1+c x+c_{2} x^{2}+c_{3} x^{3}+\ldots\right)}
$$

which can be readily evaluated numerically. The coupling $a(\tau)$ itself, where $\tau \equiv b \ln (\mu / \tilde{\Lambda})$, is obtained as the solution of the transcendental equation [6]

$$
\frac{1}{a}+c \ln \frac{c a}{1+c a}=\tau-\int_{0}^{a} d x\left[-\frac{1}{B(x)}+\frac{1}{x^{2}(1+c x)}\right],
$$

where $B(x) \equiv x^{2}\left(1+c x+c_{2} x^{2}+c_{3} x^{3}+\ldots\right)$.

\section{$3 \mathrm{RS}$ and FS dependence of the coefficients}

We first wish to parametrize the dependence of the $r_{n}$ in the coefficient function on the renormalization scheme (RS) and factorization scheme (FS).

Recall first [6] that for the single scale case of a dimensionless observable $\mathcal{R}(Q)$ with perturbation series

$$
\mathcal{R}(Q)=a+r_{1} a^{2}+r_{2} a^{3}+\ldots+r_{n} a^{n+1}+\ldots,
$$

the RS can be labelled by the non-universal coefficients of the beta-function $c_{2}, c_{3}, \ldots$,and by $\tau$, which can be traded as a parameter for $r_{1}$ since [2, 3, 6, 7]

$$
\tau-r_{1}=\rho_{0}(Q) \equiv b \ln \left(Q / \Lambda_{\mathcal{R}}\right),
$$

is an RS-invariant.Using the self-consistency of perturbation theory- that

is that the difference between a $\mathrm{N}^{n} \mathrm{LO}$ calculation (i.e up to and including 
$\left.r_{n} a^{n+1}\right)$ performed with two different RS's is $\mathrm{O}\left(a^{n+2}\right)$, one can derive expressions for the partial derivatives of the perturbative coefficients with respect to the scheme parameters. For $r_{2}$ for instance one has [6]

$$
\frac{\partial r_{2}}{\partial r_{1}}=2 r_{1}+c, \frac{\partial r_{2}}{\partial c_{2}}=-1, \frac{\partial r_{2}}{\partial c_{3}}=0, \ldots
$$

on integration one finds

$$
\begin{aligned}
r_{2}\left(r_{1}, c_{2}\right)= & r_{1}^{2}+c r_{1}+X_{2}-c_{2} \\
r_{3}\left(r_{1}, c_{2}, c_{3}\right)= & r_{1}^{3}+\frac{5}{2} c r_{1}^{2}+\left(3 X_{2}-2 c_{2}\right) r_{1}+X_{3}-\frac{1}{2} c_{3} \\
\vdots & \vdots
\end{aligned}
$$

In general the structure is

$$
r_{n}\left(r_{1}, c_{2}, \ldots, c_{n}\right)=\hat{r}_{n}\left(r_{1}, c_{2}, \ldots, c_{n-1}\right)+X_{n}-c_{n} /(n-1),
$$

where $\hat{r}_{n}$ is RG-predictable from a complete $\mathrm{N}^{n-1} \mathrm{LO}$ calculation (i.e. $r_{2}, r_{3}, \ldots, r_{n}$, and $c_{2}, c_{3}, \ldots, c_{n}$ have been computed in some RS), and the $X_{n}$ are $Q$ independent and RS-invariant constants of integration which are unknown unless a complete $\mathrm{N}^{n} \mathrm{LO}$ calculation has been performed.

As we shall see the generalization to the moment problem is a dependence $r_{n}\left(\mu, M, c_{2}, \ldots, c_{n}, d_{1}, d_{2}, \ldots, d_{n}\right)$ where the $c_{i}$ label the RS and the $d_{i}$ the FS. As before $M, \mu$ can be traded, in this case for $r_{1}(M)$ and $\tilde{r}_{1} \equiv r_{1}(M=\mu)$. There will be analogous factorization and renormalization scheme (FRS) invariants,$X_{n}$, which represent the RG-unpredictable parts of $r_{n}$. Expressions for the dependence of the coefficients on FRS parameters have been derived before in Refs. 4, 5, 8], but there were some errors in Ref. [4, in particular the dependence of $r_{2}$ on $c_{2}$ was not recognized [5]. Partially differentiating Eq.(7) with respect to $\mu, M, c_{2}, c_{3}, d_{1}, d_{2}, d_{3}$, and demanding for consistency that it be $\mathrm{O}\left(a^{4}\right)$, so that the coefficients of $a, a^{2}$ and $a^{3}$ vanish, one obtains analogous to Eqs.(12),

$$
\begin{aligned}
\mu \frac{\partial r_{1}}{\partial \mu} & =0, \quad \mu \frac{\partial r_{2}}{\partial \mu}=r_{1} b, \quad \mu \frac{\partial r_{3}}{\partial \mu}=2 r_{2} b+r_{1} b c, \\
M \frac{\partial r_{1}}{\partial M} & =d, \quad M \frac{\partial r_{2}}{\partial M}=d_{1}+d r_{1}-d L
\end{aligned}
$$




$$
\begin{aligned}
M \frac{\partial r_{3}}{\partial M} & =d_{2}+d_{1} r_{1}+d r_{2}-d r_{1} L-2 d_{1} L-d L^{2}, \\
\frac{\partial r_{1}}{\partial d_{1}} & =-\frac{1}{b}, \frac{\partial r_{2}}{\partial d_{1}}=\frac{c}{2 b}-\frac{L}{b}-\frac{r_{1}}{b}, \\
\frac{\partial r_{3}}{\partial d_{1}} & =\frac{c r_{1}}{2 b}-\frac{c^{2}}{3 b}+\frac{\left(c-r_{1}\right)}{b} L-\frac{r_{2}}{b}+\frac{c_{2}}{3 b}-\frac{L^{2}}{b}, \\
\frac{\partial r_{1}}{\partial d_{2}} & =0, \quad \frac{\partial r_{2}}{\partial d_{2}}=-\frac{1}{2 b}, \quad \frac{\partial r_{3}}{\partial d_{2}}=\frac{c}{3 b}-\frac{L}{b}-\frac{r_{1}}{2 b}, \\
\frac{\partial r_{1}}{\partial d_{3}} & =0, \quad \frac{\partial r_{2}}{\partial d_{3}}=0, \quad \frac{\partial r_{3}}{\partial d_{3}}=-\frac{1}{3 b}, \\
\frac{\partial r_{1}}{\partial c_{2}} & =0, \quad \frac{\partial r_{2}}{\partial c_{2}}=\frac{3 d}{2 b}, \quad \frac{\partial r_{3}}{\partial c_{2}}=\frac{4 d_{1}}{3 b}+3 \frac{d L}{b}+3 \frac{d r_{1}}{2 b}-r_{1}-5 \frac{c d}{3 b}, \\
\frac{\partial r_{1}}{\partial c_{3}} & =0, \quad \frac{\partial r_{2}}{\partial c_{3}}=0, \quad \frac{\partial r_{3}}{\partial c_{3}}=\frac{5 d}{6 b} .
\end{aligned}
$$

Here we have defined for convenience $L \equiv b \ln (M / \mu)$. Consistently integrating the partial derivatives of $r_{1}$ yields

$$
r_{1}=\frac{d}{b} \tau_{M}-\frac{d_{1}}{b}-X_{1}(Q)
$$

where $\tau_{M} \equiv b \ln (M / \tilde{\Lambda})$ and $X_{1}(Q)$ is an FRS-invariant, analogous to $\rho_{0}(Q)$ for the single scale problem defined in Eq.(11). Exactly analogous to $\Lambda_{\mathcal{R}}$, for the moment problem one can define an FRS-invariant $\Lambda_{\mathcal{M}}$ so that

$$
\frac{d}{b} \tau_{M}-\frac{d_{1}}{b}-r_{1}=X_{1}(Q) \equiv d \ln \left(\frac{Q}{\Lambda_{\mathcal{M}}}\right)
$$

Consistently integrating the remaining partial derivatives and using Eq.(16) to recast the $M$ and $\mu$ dependence in terms of $r_{1}$ and $\tilde{r}_{1}$, one obtains the explicit dependence of $r_{2}$ and $r_{3}$ on the FRS parameters $r_{1}, \tilde{r}_{1}, d_{1}, d_{2}, d_{3}, c_{2}, c_{3}$

$$
\begin{aligned}
r_{2}= & \left(\frac{1}{2}-\frac{b}{2 d}\right) r_{1}^{2}+\frac{b}{d} r_{1} \tilde{r}_{1}+\frac{c d_{1}}{2 b}-\frac{d_{2}}{2 b}-\frac{d c_{2}}{2 b}+X_{2} \\
r_{3}= & \left(\frac{b^{2}}{d^{2}}-\frac{3 b}{2 d}+\frac{1}{2}\right) \frac{r_{1}^{3}}{3}+\left(-\frac{b^{2}}{d^{2}}+\frac{b}{d}\right) r_{1}{ }^{2} \tilde{r}_{1}+\left(\frac{b c}{d}+\frac{2 b d_{1}}{d^{2}}\right) r_{1} \tilde{r}_{1} \\
& +\left(-\frac{b c}{2 d}-\frac{b d_{1}}{d^{2}}+\frac{d_{1}}{d}\right) r_{1}{ }^{2}+\left(-\frac{d c_{2}}{2 b}+\frac{c d_{1}}{2 b}+X_{2}+\frac{d_{1}{ }^{2}}{2 d b}+\frac{d_{2}}{d}-\frac{d_{2}}{2 b}-c_{2}\right) r_{1}
\end{aligned}
$$




$$
\begin{aligned}
& +\left(\frac{d_{1}^{2}}{d^{2}}-\frac{d_{2}}{d}+\frac{c d_{1}}{d}+\frac{2 b X_{2}}{d}\right) \tilde{r}_{1}+\frac{b^{2}}{d^{2}} r_{1} \tilde{r}_{1}^{2}+\left(-\frac{d_{1} c^{2}}{3 b}+\frac{2 d_{1} X_{2}}{d}\right. \\
& \left.+\frac{d_{1}^{3}}{3 b d^{2}}+\frac{d c c_{2}}{3 b}+\frac{c d_{1}^{2}}{2 d b}+\frac{d_{3}}{3 d}-\frac{d c_{3}}{6 b}-\frac{2 d_{1} c_{2}}{3 b}+\frac{d_{2} c}{3 b}+X_{3}\right) \\
& \quad \vdots,
\end{aligned}
$$

analogous to Eqs.(13) in the single scale case. Notice that we could equally use $r_{1}$ and $L$ as parameters instead of $r_{1}$ and $\tilde{r}_{1}$, since $L=(b / d)\left(r_{1}-\tilde{r}_{1}\right)$. As in the single scale case there are constants of integration $X_{n}$ representing the RG-unpredictable part of $r_{n}$. They are $Q$-independent and FRS-invariant.

In the single scale case parametrizing the RS-dependence using $r_{1}, c_{2}, c_{3}, \ldots$ means that given a complete $\mathrm{N}^{n} \mathrm{LO}$ calculation $X_{2}, X_{3}, \ldots, X_{n}$ will be known. Using Eqs.(13) to sum to all-orders the RG-predictable terms, i.e. those not involving $X_{n+1}, X_{n+2}, \ldots$, with coupling $a\left(r_{1}, c_{2}, c_{3}, \ldots\right)$ is equivalent to $\mathrm{N}^{n} \mathrm{LO}$ perturbation theory in the scheme with $r_{1}=c_{2}=c_{3}=\ldots=0$, and yields the sum

$$
\mathcal{R}^{(n)}=a_{0}+X_{2} a_{0}{ }^{2}+X_{3} a_{0}^{3}+\ldots+X_{n} a_{0}{ }^{n},
$$

where $a_{0} \equiv a(0,0,0, \ldots)$ is the coupling in this scheme. From Eqs.(9) and (11) it satisfies

$$
\frac{1}{a_{0}}+c \ln \left(\frac{c a_{0}}{1+c a_{0}}\right)=b \ln \left(\frac{Q}{\Lambda_{\mathcal{R}}}\right) .
$$

In fact the solution of this transcendental equation can be written in closed form in terms of the Lambert $W$-function [9, 10], defined implicitly by $W(z) \exp (W(z))=z$

$$
\begin{aligned}
a_{0} & =-\frac{1}{c[1+W(z(Q))]} \\
z(Q) & \equiv-\frac{1}{e}\left(\frac{Q}{\Lambda_{\mathcal{R}}}\right)^{-b / c} .
\end{aligned}
$$

A similar expansion to Eq.(19), but motivated differently, has been suggested in Ref. [11].

In the moment problem by an exactly similar argument, with the chosen parametrization of FRS, given a complete $\mathrm{N}^{n} \mathrm{LO}$ calculation (i.e. a calculation of $r_{1}, r_{2}, \ldots, r_{n}$ and the $d_{1}, d_{2}, \ldots, d_{n}$ and $c_{2}, c_{3}, \ldots, c_{n}$ in some FRS) the 
invariants $X_{2}, X_{3}, \ldots, X_{n}$ will be known. Using Eqs.(18) to sum to all-orders the RG-predictable terms not involving $X_{n+1}, X_{n+2}, \ldots$, will be equivalent to working with an FRS in which all the FRS parameters are set to zero. $\tilde{r}_{1}=0$ means that $\mu=M$. Setting $r_{1}=0, d_{1}=0$ in Eq.(17) yields $\tau_{M}=b \ln \left(Q / \Lambda_{\mathcal{M}}\right)$, so that $a=\tilde{a}=a_{0}$, given by Eq.(21) with $\Lambda_{\mathcal{R}}$ replaced by $\Lambda_{\mathcal{M}}$. Further, with $c_{i}=d_{i}=0$ the integral $\mathcal{I}(a)$ in Eq.(8) vanishes, so that finally the sum of all RG-predictable terms for the moment problem at $\mathrm{N}^{n} \mathrm{LO}$ will be

$$
\mathcal{M}=A\left(\frac{c a_{0}}{1+c a_{0}}\right)^{d / b}\left(1+X_{2} a_{0}^{2}+X_{3} a_{0}{ }^{3}+\ldots+X_{n} a_{0}{ }^{n}\right),
$$

with an extremely similar structure to the single scale case in Eq.(19). Substituting for $a_{0}$ in terms of the Lambert $W$-function using Eq.(21) we then obtain

$$
\begin{aligned}
\mathcal{M} & =A[-W(z(Q))]^{b / d}\left(1+X_{2} a_{0}{ }^{2}+\ldots\right) \\
z(Q) & \equiv-\frac{1}{e}\left(\frac{Q}{\Lambda_{\mathcal{M}}}\right)^{-b / c} .
\end{aligned}
$$

So that moments of structure functions have a $Q$-dependence naturally involving a power of the Lambert $W$-function.

As stressed in Ref.[1] the result of resumming all RG-predictable terms depends on the chosen parametrization of RS. By simply translating the parameters to a new set $\breve{r}_{1}=r_{1}-\bar{r}_{1}, \breve{c}_{2}=c_{2}-\bar{c}_{2}, \ldots$ etc., where the barred quantities are constants, one finds corresponding new constants of integration $\breve{X}_{n}$. The result of resumming all RG-predictable terms with this new parametrization then corresponds to standard fixed-order perturbation theory in the RS with $r_{1}=\bar{r}_{1}, c_{2}=\bar{c}_{2}, \ldots$, or equivalently with $\breve{r}_{1}=\breve{c}_{2}=\breve{c}_{3}=\ldots=0$. The key point is that $r_{1}$ has a special status since it contains the ultraviolet (UV) logarithms which build the physical $Q$-dependence of $\mathcal{R}(Q)$. Standard RG-improvement corresponds to shifting the parameter $r_{1}$, in which case the resulting constants of integration $\breve{X}_{n}$ contain physical UV logarithms which are not all resummed. Thus $r_{1}$ should be used as the parameter. An exactly similar statement holds for $r_{1}$ and $\tilde{r}_{1}$ in the moment problem. We shall identify the UV logarithms and show how their complete resummation builds the correct physical $Q$-dependence in the next section. 
We shall refer to the expansions in Eqs.(19) and (22) as Complete RGimproved (CORGI) results. Whilst the parameters implicitly containing the UV logarithms do have a special status, the remaining dimensionless parameters $c_{i}$ and $d_{i}$ can be reparametrized as one pleases. As an example, in the Effective Charge approach of Grunberg [2, 3] one chooses $\bar{c}_{2}, \bar{c}_{3}, \ldots, \bar{c}_{n}$ so that $\breve{X}_{2}, \breve{X}_{3}, \ldots, \breve{X}_{n}$ are all zero at $\mathrm{N}^{n} \mathrm{LO}$, corresponding to $r_{1}=r_{2}=\ldots=r_{n}=0$, and this is a priori equally reasonable. In the moment problem one can correspondingly choose the $\bar{c}_{i}$ and $\bar{d}_{i}$ so that at $\mathrm{N}^{n} \mathrm{LO}$ the $\breve{X}_{i}$ all vanish and $r_{1}=r_{2}=\ldots=r_{n}=0$. If one further demands that the integral $\mathcal{I}(a)$ in Eq.(8) vanishes order-by-order in a a unique FRS is selected in which moments have the form

$$
\mathcal{M}=A\left(\frac{c \hat{\mathcal{R}}}{1+c \hat{\mathcal{R}}}\right)^{d / b}
$$

Where $\hat{\mathcal{R}}$ is an effective charge which has a perturbation series of the form,

$$
\hat{\mathcal{R}}=a+\hat{r}_{1} a^{2}+\hat{r}_{2}+\ldots+\hat{r}_{n} a^{n+1}+\ldots
$$

This is similar to Grunberg's proposal [3] to associate an effective charge with $\mathcal{M}$ so that $\mathcal{M}=A(c \hat{\mathcal{R}})^{d / b}$. The $\hat{r}_{i}$ are built from the $c_{i}, d_{i}, M$ and $\mu$,and are RS-dependent, but FS-independent. Effectively $\hat{\mathcal{R}}$ can be RG-improved as in the single scale case. We have for instance

$$
\hat{r}_{1}=b \ln (\mu / \tilde{\Lambda})-b \ln (M / \tilde{\Lambda})-\frac{b}{d} r_{1}+d_{1} / d=\tau-X_{1}(Q)
$$

where we have used Eq.(17) . Comparing with Eq.(11) we see that treating $\hat{\mathcal{R}}$ as a single scale problem we have $\rho_{0}(Q)=X_{1}(Q)$. This further implies that $\Lambda_{\hat{\mathcal{R}}}=\Lambda_{\mathcal{M}}$ and so the corresponding CORGI couplings are identical. The CORGI expansion for $\hat{\mathcal{R}}$ will be of the form

$$
\hat{\mathcal{R}}=a_{0}+\hat{X}_{2} a_{0}^{2}+\hat{X}_{3} a_{0}^{3}+\ldots
$$

Inserting this result in Eq.(24) and re-expanding in $a_{0}$ will reproduce the CORGI expansion in Eq.(22). 


\section{Complete RG-improvement}

In the single scale case using Eq.(11) one can write

$$
r_{1}=b\left(\ln \frac{\mu}{\tilde{\Lambda}}-\ln \frac{Q}{\Lambda_{\mathcal{R}}}\right)
$$

The first $\mu$-dependent logarithm depends on the RS, whereas the second $Q$-dependent UV logarithm will generate the physical $Q$-dependence and is RS-invariant. If one makes the simplification that $c=0$ and sets $c_{2}=c_{3}=$ $\ldots=0$, then the coupling is given by

$$
a(\mu)=1 / b \ln \left(\frac{\mu}{\tilde{\Lambda}}\right) .
$$

The sum to all-orders of the RG-predictable terms from Eqs.(13), given a NLO calculation of $r_{1}$, simplifies to a geometric progression,

$$
\mathcal{R}=a+r_{1} a+r_{1}^{2} a^{3}+\ldots+r_{1}^{n} a^{n+1}+\ldots .
$$

The idea of complete RG-improvement is that dimensionful renormalization scales, in this case $\mu$, should be held strictly independent of the physical energy scale $Q$ on which $\mathcal{R}(Q)$ depends. In this way the $Q$-dependence is built entirely by the "physical" UV logarithms $b \ln \left(Q / \Lambda_{\mathcal{R}}\right)$ contained in $r_{1}$, and the convention-dependent logarithms of $\mu$ cancel between $a(\mu)$ and $r_{1}(\mu)$ , when the all-orders sum in Eq.(30) is evaluated. The conventional fixedorder NLO truncation $\mathcal{R}=a(\mu)+r_{1}(\mu) a(\mu)^{2}$, only makes sense if $\mu=x Q$, but then the resulting $Q$-dependence involves the arbitrary parameter $x$. In contrast using Eqs.(28),(29) and summing the geometric progression in Eq.(30) gives,

$$
\mathcal{R}(Q) \approx a(\mu) /\left[1-\left(b \ln \frac{\mu}{\tilde{\Lambda}}-b \ln \frac{Q}{\Lambda_{\mathcal{R}}}\right) a(\mu)\right]=1 / b \ln \left(Q / \Lambda_{\mathcal{R}}\right),
$$

correctly reproducing the large- $Q$ behaviour of $\mathcal{R}(Q)$,

$$
\mathcal{R}(Q) \approx 1 / b \ln \left(Q / \Lambda_{\mathcal{R}}\right)+O\left(1 / b \ln \left(Q / \Lambda_{\mathcal{R}}\right)^{3} .\right.
$$

In the moment problem the analogous $\mathrm{UV}$ logarithm is $b \ln \left(Q / \Lambda_{\mathcal{M}}\right)$ introduced in Eq.(17), and analogous to Eq.(28) we will have

$$
r_{1}=d\left(\ln \frac{M}{\tilde{\Lambda}}-\ln \frac{Q}{\Lambda_{\mathcal{M}}}\right)-\frac{d_{1}}{b} .
$$


Given a NLO calculation of $r_{1}$ we wish to see how the physical $Q$-dependence of $\mathcal{M}(Q)$ arises on resumming to all-orders the UV logarithms contained in the RG-predictable terms from Eqs.(18). If we make similar approximations, so that $c=0$ and the $d_{i}$ and $c_{i}$ are set to zero, then

$$
\mathcal{M}=A(c a(M))^{d / b}\left(1+r_{1} a(\mu)+r_{2} a(\mu)^{2}+\ldots\right) .
$$

We retain the overall factor of $c^{d / b}$. The task is then to show that on resumming the RG-predictable terms in the coefficient function to all-orders the $\ln (M / \tilde{\Lambda})$ and $\ln (\mu / \tilde{\Lambda})$ contained in $r_{1}$ and $\tilde{r}_{1}$ cancel with those in the couplings $a(M)$ and $a(\mu)$ to yield the physical Q-dependence

$$
\mathcal{M}(Q) \approx A c^{d / b}\left(1 / b \ln \left(Q / \Lambda_{\mathcal{M}}\right)\right)^{d / b}\left(1+O\left(1 / \ln \left(Q / \Lambda_{\mathcal{M}}\right)\right)^{2}\right) .
$$

Again, the complete RG-improvement summing over all UV logarithms is forced on one if $\mu$ and $M$ are held independent of $Q$.

The algebraic structure of the resummation of RG-predictable terms for the moment problem is considerably more complicated than the geometric progression of Eq.(30) encountered in the single scale case. With the simplifications $c=0, c_{i}=0, d_{i}=0$ the first two RG-predictable coefficients from $\operatorname{Eqs}(18)$ are

$$
\begin{aligned}
& r_{2}=\left(\frac{1}{2}-\frac{b}{2 d}\right) r_{1}^{2}+\frac{b}{d} r_{1} \tilde{r_{1}} \\
& r_{3}=\left(\frac{b^{2}}{d^{2}}-\frac{3 b}{2 d}+\frac{1}{2}\right) \frac{r_{1}^{3}}{3}+\left(\frac{-b^{2}}{d^{2}}+\frac{b}{d}\right) r_{1}{ }^{2} \tilde{r_{1}}+\frac{b^{2}}{d^{2}} r_{1}{\tilde{r_{1}}}^{2}
\end{aligned}
$$

Suitably generalizing the partial derivatives in Eqs.(15) one can arrive at a general form for the RG-predictable terms. It is useful to arrange them in columns,

$$
\left(\begin{array}{cccc}
r_{1} \rightarrow\left(\frac{b}{d} \tilde{r_{1}}\right)^{0} r_{1} \tilde{a} & 0 & 0 & \ldots \\
r_{2} \rightarrow\left(\frac{b}{d} \tilde{r_{1}}\right)^{1} r_{1} \tilde{a}^{2} & \left(1-\frac{b}{d}\right) \frac{r_{1}^{2}}{2} \tilde{a}^{2} & 0 & \ldots \\
r_{3} \rightarrow\left(\frac{b}{d} \tilde{r_{1}}\right)^{2} r_{1} \tilde{a}^{3} & 2\left(\frac{b}{d} \tilde{r_{1}}\right)\left(1-\frac{b}{d}\right) \frac{r_{1}^{2}}{2} \tilde{a}^{3} & \left(1-\frac{b}{d}\right)\left(\frac{1}{2}-\frac{b}{d}\right) \frac{r_{1}^{3}}{3} \tilde{a}^{3} & \ldots \\
r_{4} \rightarrow\left(\frac{b}{d} \tilde{r_{1}}\right)^{3} r_{1} \tilde{a}^{4} & 3\left(\frac{b}{d} \tilde{r_{1}}\right)^{2}\left(1-\frac{b}{d}\right) \frac{r_{1}^{2}}{2} \tilde{a}^{4} & 3\left(\frac{b}{d} \tilde{r_{1}}\right)\left(1-\frac{b}{d}\right)\left(\frac{1}{2}-\frac{b}{d}\right) \frac{r_{1}}{3} \tilde{a}^{4} & \ldots \\
r_{5} \rightarrow\left(\frac{b}{d} \tilde{r_{1}}\right)^{4} r_{1} \tilde{a}^{5} & 4\left(\frac{b}{d} \tilde{r_{1}}\right)^{3}\left(1-\frac{b}{d}\right) \frac{r_{1}^{2}}{2} \tilde{a}^{5} & 6\left(\frac{b}{d} \tilde{r}_{1}\right)^{2}\left(1-\frac{b}{d}\right)\left(\frac{1}{2}-\frac{b}{d}\right) \frac{r_{1}}{3} \tilde{a}^{5} & \ldots \\
\vdots & \vdots & \vdots & \ddots
\end{array}\right)
$$


The idea will be to resum each column separately. Denoting the sum of the $\mathrm{m}^{\text {th }}$ column by $S_{m}$, one finds

$$
\begin{aligned}
S_{1} & =r_{1} \tilde{a}+\left(\frac{b}{d} \tilde{r_{1}}\right) r_{1} \tilde{a}^{2}+\left(\frac{b}{d} \tilde{r_{1}}\right)^{2} r_{1} \tilde{a}^{3}+\left(\frac{b}{d} \tilde{r_{1}}\right)^{3} r_{1} \tilde{a}^{4}+\left(\frac{b}{d} \tilde{r}_{1}\right)^{4} r_{1} \tilde{a}^{5}+\ldots \\
& =r_{1} \tilde{a}\left[1+\left(\frac{b}{d} \tilde{r_{1}} \tilde{a}\right)+\left(\frac{b}{d} \tilde{r_{1}} \tilde{a}\right)^{2}+\left(\frac{b}{d} \tilde{r_{1}} \tilde{a}\right)^{3}+\left(\frac{b}{d} \tilde{r_{1}} \tilde{a}\right)^{4}+\ldots\right] \\
& =r_{1} \tilde{a}\left(1-\frac{b}{d} \tilde{r_{1}} \tilde{a}\right)^{-1}
\end{aligned}
$$

Careful examination of the pattern of terms in Eq.(38) leads to the general result for $S_{m}$ for $m>1$,

$$
S_{m}=(-1)^{2 m-1}\left(\frac{b}{d}-1\right)\left(\frac{b}{d}-\frac{1}{2}\right)\left(\frac{b}{d}-\frac{1}{3}\right)+\ldots+\left(\frac{b}{d}-\frac{1}{m-1}\right) \frac{S_{1}{ }^{m}}{m}
$$

Finally the resummed RG-predictable terms in the coefficient function will follow from $\mathcal{C}=1+S_{1}+S_{2}+S_{3}+\ldots+S_{n}+\ldots$ Introducing for convenience $x \equiv S_{1}=r_{1} \tilde{a}\left(1-\frac{b}{d} \tilde{r}_{1} \tilde{a}\right)^{-1}$, we find

$$
\begin{aligned}
\mathcal{C} & =1+x-\left(\frac{b}{d}-1\right) \frac{x^{2}}{2}+\left(\frac{b}{d}-1\right)\left(\frac{b}{d}-\frac{1}{2}\right) \frac{x^{3}}{3}-\left(\frac{b}{d}-1\right)\left(\frac{b}{d}-\frac{1}{2}\right)\left(\frac{b}{d}-\frac{1}{3}\right) \frac{x^{4}}{4}+\ldots \\
& =1+\frac{d}{b}\left(\frac{b x}{d}\right)+\frac{1}{2 !} \frac{d}{b}\left(\frac{d}{b}-1\right)\left(\frac{b x}{d}\right)^{2}++\frac{1}{3 !} \frac{d}{b}\left(\frac{d}{b}-1\right)\left(\frac{d}{b}-2\right)\left(\frac{b x}{d}\right)^{3}+\ldots \\
& =\left(1+\frac{b}{d} x\right)^{d / b} .
\end{aligned}
$$

Substituting for $x$ yields

$$
\mathcal{C}=\left\{1+\frac{b}{d}\left[r_{1} \tilde{a}\left(1-\frac{b}{d} \tilde{r_{1}} \tilde{a}\right)^{-1}\right]\right\}^{\frac{d}{b}}=\left[\frac{1-\frac{b}{d} \tilde{r_{1}} \tilde{a}+\frac{b}{d} r_{1} \tilde{a}}{1-\frac{b}{d} \tilde{r_{1}} \tilde{a}}\right]^{\frac{d}{b}}
$$

We can write the numerator in Eq.(42) as

$$
\left(1-\frac{b}{d} \tilde{r_{1}} \tilde{a}+\frac{b}{d} r_{1} \tilde{a}\right)=\left[1+\tilde{a} b\left(\frac{r_{1}-\tilde{r_{1}}}{d}\right)\right]=(1+\tilde{a} L)
$$

Where $L=b \ln (M / \mu)=b\left(r_{1}-\tilde{r}_{1}\right) / d$. Since we are setting $c=c_{2}=c_{3}=$ $\ldots=0$ one has $(1+\tilde{a} L)^{-1}=a / \tilde{a}$, substituting this into Eq.(42) gives

$$
\mathcal{C}=\left[\left(1-\frac{b}{d} \tilde{r_{1}} \tilde{a}\right) \frac{a}{\tilde{a}}\right]^{\frac{-d}{b}}=\left[\frac{\left(1-\frac{b}{d} \tilde{r_{1}} \tilde{a}\right)}{\tilde{a}} a\right]^{\frac{-d}{b}}
$$


Since $\tilde{a}=a(\mu)=1 / \tau$ we can rearrange Eq.(16) to obtain

$$
\tilde{r_{1}}=\frac{d}{b} \frac{1}{\tilde{a}}-d \ln \frac{Q}{\Lambda_{\mathcal{M}}}
$$

and substituting this result into Eq.(43) we find

$$
\mathcal{C}=\left(\frac{1}{b \ln \left(Q / \Lambda_{\mathcal{M}}\right)}\right)^{d / b} a^{-d / b}
$$

Combining this with the anomalous dimension part $(c a)^{d / b}$ we reproduce the physical $Q$-dependence of $\mathcal{M}(Q)$ in Eq.(35).

\section{Discussion and Conclusions}

An alternative and more straightforward way of understanding the CORGI proposal is as follows. Given a dimensionless observable $\mathcal{R}(Q)$, dependent on the single dimensionful scale $Q$, we clearly must have, on grounds of generalized dimensional analysis [12]

$$
\mathcal{R}(Q)=\Phi\left(\frac{\Lambda}{Q}\right)
$$

where $\Lambda$ is a dimensionful scale, connected with the universal dimensional transmutation parameter of the theory, whose definition will depend on the way in which ultraviolet divergences are removed, $\Lambda_{\overline{M S}}$ for instance. We can try to invert Eq.(47) to obtain

$$
\frac{\Lambda}{Q}=\Phi^{-1}(\mathcal{R}(Q))
$$

where $\Phi^{-1}$ is the inverse function. This is indeed the basic motivation for Grunberg's Effective Charge approach [2, 3]. We are assuming massless quarks here. The extension if one includes masses has been discussed in [3], 13]. The structure of $\Phi^{-1}$ is [14, 15]

$$
\mathcal{F}(\mathcal{R}(Q)) \mathcal{G}(\mathcal{R}(Q))=\Lambda_{\mathcal{R}} / Q
$$


where

$$
\mathcal{F}(\mathcal{R}(Q)) \equiv e^{-1 / b \mathcal{R}}(1+1 / b \mathcal{R})^{c / b}
$$

is a universal function of $\mathcal{R} . \Lambda_{\mathcal{R}}$ is connected with the universal parameter $\Lambda_{\overline{M S}}$ by the relation

$$
\Lambda_{\mathcal{R}}=e^{r / b} \tilde{\Lambda}_{\overline{M S}},
$$

which follows from Eq.(11), with $r \equiv r_{1} \overline{M S}(\mu=Q)$ the NLO $\overline{M S}$ coefficient. Note that $r$ is $Q$-independent. The tilde over $\Lambda$ reflects the convention assumed in integrating the beta-function equation to obtain Eq.(9) [6], and $\tilde{\Lambda}_{\overline{M S}}=(2 c / b)^{-c / b} \Lambda_{\overline{M S}}$ in terms of the standard convention . The function $\mathcal{G}(\mathcal{R}(Q))$ has the expansion

$$
\mathcal{G}(\mathcal{R}(Q))=1-\frac{X_{2}}{b} \mathcal{R}(Q)+O\left(\mathcal{R}^{2}\right)+\ldots
$$

Here $X_{2}$ is the NNLO RS-invariant constant of integration which arises in Eqs.(13). Assembling all this we finally obtain the desired inverse relation between $\mathcal{R}$ and $\Lambda$, the universal dimensional transmutation parameter of the theory

$$
Q \mathcal{F}(\mathcal{R}(Q)) \mathcal{G}(\mathcal{R}(Q)) e^{-r / b}(2 c / b)^{c / b}=\Lambda_{\overline{M S}} .
$$

Notice that all dependence on the subtraction scheme chosen resides in the single factor $e^{-r / b}$, the remainder of the expression being independent of this choice. This corresponds to the observation of Celmaster and Gonsalves [16], that $\Lambda$ 's with different subtraction conventions can be exactly related given a one-loop (NLO) calculation. If only a NLO calculation has been performed $\mathcal{G}=1$ since $X_{2}$ will be unknown, so that the best one can do in reconstructing $\Lambda_{\overline{M S}}$ is

$$
Q \mathcal{F}(\mathcal{R}(Q)) e^{-r / b}(2 c / b)^{c / b}=\Lambda_{\overline{M S}} .
$$

This is precisely the result obtained on inverting the NLO CORGI result $\mathcal{R}=a_{0}$ given by Eq.(21).

The essential point is that the dimensional transmutation scale $\Lambda$ is the fundamental object. In contrast the convention-dependent dimensionful scales $\mu$ and $M$ are ultimately irrelevant quantities which cancel out of physical predictions if one takes care to resum all of the ultraviolet logarithms that build the physical $Q$-dependence in association with $\Lambda$. Our purpose 
has been to indicate that the unphysical $\mu$ and $M$ dependence of conventional fixed-order perturbation theory reflects its failure to resum all of these RG-predictable terms. We have analyzed how Eq.(54) is built by explicitly resumming the convention-dependent logarithms together with the ultraviolet logarithms. Having done this, however, one can simply use Eq.(53) to test perturbative QCD. Given at least a NLO calculation for an observable $\mathcal{R}(Q)$ one simply substitutes the data values into Eq. (53), where $\mathcal{G}(\mathcal{R}(\mathcal{Q})$ ) can include NNLO and higher corrections if known, and obtains $\Lambda_{\overline{M S}}$. To the extent that remaining higher-order perturbative and possible power corrections are small, one should find consistent values of $\Lambda_{\overline{M S}}$ for different observables. There is no need to mention $\mu$ or $M$ in this analysis, let alone to vary them over an ad hoc range of values. For the moment problem the result coresponding to Eq.(53) is

$$
Q \overline{\mathcal{F}}\left(\frac{\mathcal{M}}{A}\right) \overline{\mathcal{G}}\left(\frac{\mathcal{M}}{A}\right) e^{-\hat{r} / b}(2 c / b)^{c / b}=\Lambda_{\overline{M S}},
$$

where $\hat{r} \equiv \hat{r}_{1}^{\overline{M S}}(\mu=Q)$ is defined in Eq. (26). The modified functions $\overline{\mathcal{F}}$ and $\overline{\mathcal{G}}$ are most easily obtained by noting that $\hat{\mathcal{R}}$ in Eq.(24) is directly related to $\mathcal{M} / A$ and also satisfies Eq.(53). One finds

$$
\begin{aligned}
& \overline{\mathcal{F}}(x)=\exp \left[b c\left(1-x^{-b / d}\right]\left(1+b c\left(x^{-b / d}-1\right)\right)^{c / b}\right. \\
& \overline{\mathcal{G}}(x)=\left(1-\frac{X_{2}}{d} \frac{x^{b / d}}{c\left(1-x^{b / d}\right)}+\ldots\right) .
\end{aligned}
$$

Where $X_{2}$ is the NNLO FRS-invariant which arises in Eqs.(18). The schemeindependent parameter $A$ reflects a physical property of the operator $\mathcal{O}_{n}$ in Eq.(2). $A_{n}$ and $\Lambda_{\overline{M S}}$ should be fitted simultaneously to the data for $\mathcal{M}_{n}(Q)$ using Eq.(55).

We hope to report direct fits of data to $\Lambda_{\overline{M S}}$ as outlined above, for both $e^{+} e^{-}$jet observables [17] and structure functions and their moments [18], in future publications.

\section{Aknowledgements}

A.M. acknowledges the financial support of the Iranian government and also thanks S.J. Burby for useful discussions. 


\section{References}

[1] C.J. Maxwell, hep-ph/9908463.

[2] G. Grunberg, Phys. Lett. B95 (1980) 70.

[3] G. Grunberg, Phys. Rev. D29 (1984) 2315.

[4] H. David Politzer, Nucl Phys B194 (1982) 493.

[5] P.M. Stevenson and H. David Politzer, Nucl. Phys. B277 (1986) 758.

[6] P.M. Stevenson, Phys. Rev. D23 (1984) 2916.

[7] A.L. Kataev, N.V. Krasnikov, and A.A. Pivovarov, Nucl. Phys B198 (1982) 508 .

[8] J. Chyla, Nucl. Phys. B321 (1989) 374.

[9] B. Magradze, hep-ph/9808247 and hep-ph/9911456.

[10] Einan Gardi, Georges Grunberg and Marek Karliner, JHEP 07 (1998) 007.

[11] D.S. Kourashev, hep-ph/99112410.

[12] P.M. Stevenson, Ann. Phys. 152 (1981) 383.

[13] V.Gupta, D.V. Shirkov, and O.V. Tarasov, Int. J. Mod. Phys. A6 (1991) 338.

[14] D.T. Barclay, C.J. Maxwell and M.T. Reader, Phys. Rev.D49 (1994) 3480 .

[15] C.J. Maxwell, Phys. Lett. B409 (1997) 450.

[16] W. Celmaster and R.J. Gonsalves, Phys. Rev. D20 (1979) 1420.

[17] S.J. Burby and C.J. Maxwell, in preparation.

[18] C.J. Maxwell and A. Mirjalili, in preparation. 